\title{
Chromosomal imbalance in the progression of high-risk non-muscle invasive bladder cancer
}

\author{
Karsten Zieger*1,2, Carsten Wiuf33, Klaus Møller-Ernst Jensen², \\ Torben Falck Ørntoft ${ }^{1}$ and Lars Dyrskjøt ${ }^{1}$
}

\author{
Address: ${ }^{1}$ Department of Molecular Medicine, Aarhus University Hospital Skejby, Aarhus N, Denmark, ${ }^{2}$ Department of Urology, Aarhus University \\ Hospital Skejby, Aarhus N, Denmark and ${ }^{3}$ Bioinformatics Research Center, Aarhus University, Aarhus, Denmark \\ Email: Karsten Zieger* - karsten.zieger@ki.au.dk; Carsten Wiuf - wiuf@birc.au.dk; Klaus Møller-Ernst Jensen - klaujens@rm.dk; \\ Torben Falck Ørntoft - orntoft@ki.au.dk; Lars Dyrskjøt - lars@ki.au.dk \\ * Corresponding author
}

Published: 16 May 2009

BMC Cancer 2009, 9:149 doi:10.1186/147/-2407-9-149
Received: 15 September 2008

Accepted: 16 May 2009

This article is available from: http://www.biomedcentral.com/I47I-2407/9//49

(C) 2009 Zieger et al; licensee BioMed Central Ltd.

This is an Open Access article distributed under the terms of the Creative Commons Attribution License (http://creativecommons.org/licenses/by/2.0), which permits unrestricted use, distribution, and reproduction in any medium, provided the original work is properly cited.

\begin{abstract}
Background: Non-muscle invasive bladder neoplasms with invasion of the lamina propria (stage $\mathrm{TI}$ ) or high grade of dysplasia are at "high risk" of progression to life-threatening cancer. However, the individual course is difficult to predict. Chromosomal instability $(\mathrm{Cl})$ is associated with high tumor stage and grade, and possibly with the risk of progression.
\end{abstract}

Methods: To investigate the relationship between $\mathrm{Cl}$ and subsequent disease progression, we performed a casecontrol-study of 125 patients with "high-risk" non-muscle invasive bladder neoplasms, 67 with later disease progression, and 58 with no progression. Selection criteria were conservative (non-radical) resections and full prospective clinical follow-up ( $>5$ years). We investigated primary lesions in 59, and recurrent lesions in 66 cases.

We used Affymetrix GeneChip ${ }^{\circledR}$ Mapping $10 \mathrm{~K}$ and $50 \mathrm{~K} \mathrm{SNP}$ microarrays to evaluate genome wide chromosomal imbalance (loss-of-heterozygosity and DNA copy number changes) in 48 representative tumors. DNA copy number changes of 15 key instability regions were further investigated using QPCR in I0I tumors (including 25 tumors also analysed on $50 \mathrm{~K}$ SNP microarrays).

Results: Chromosomal instability did not predict any higher risk of subsequent progression. Stage TI and highgrade tumors had generally more unstable genomes than tumors of lower stage and grade (mostly non-primary tumors following a "high-risk" tumor). However, about $25 \%$ of the "high-risk" tumors had very few alterations. This was independent of subsequent progression. Recurrent lesions represent underlying field disease. A separate analysis of these lesions did neither reflect any difference in the risk of progression. Of specific chromosomal alterations, a possible association between loss of chromosome $8 \mathrm{pll}$ and the risk of progression was found. However, the predictive value was limited by the heterogeneity of the changes.

Conclusion: Chromosomal instability $(\mathrm{Cl})$ was associated with "high risk" tumors (stage TI or high-grade), but did not predict subsequent progression. Recurrences after "high-risk" tumors had fewer chromosomal alterations, but there was no association with the risk of progression in this group either. Thus, the prediction of progression of "high risk" non-muscle invasive bladder tumors using chromosomal changes is difficult. Loss of chromosome $8 \mathrm{pll}$ may play a role in the progression process. About $25 \%$ of the "high risk" tumors were chromosomal stable. 


\section{Background}

The individual course of non-muscle invasive bladder neoplasm is difficult to predict. In particular, tumors with invasion of the lamina propria (stage T1) or high grade of dysplasia have a $30-60 \%$ risk of progression to muscle invasive, life-threatening cancer $[1,2]$. A transurethral, bladder-sparing approach is often chosen to cure nonmuscle invasive tumors. However, recurrence is common $[2,3]$. Remnants of tumor tissue due to incomplete resection would be the source of true tumor recurrence at the original site. New tumor occurrences at distant sites, however, are owed to cancer precursors in the mucosa - socalled field disease - a very common condition in bladder cancer. Multifocality or concomitant carcinoma in situ (CIS), a highly dysplastic flat precursor lesion, may be indicators of field disease. The evaluation of the malignant potential of field disease is of particular clinical interest.

The assessment of molecular changes may improve this evaluation. The hitherto best known molecular event predictive of advanced malignant development is the loss of p53 function, which is found in the majority of progressed bladder cancers and, notably, in CIS [4,5]. Several mechanisms may lead to loss of p53 function, e.g. gene mutation or loss, transcriptional downregulation and enhanced degradation. P53 is part of the complex DNA damage control system, which surveys the integrity of the genome. Dysfunction of this system leads to chromosomal instability (CI), a key event of malignant tumor development [6]. CI is easier to assess and to interpret than loss of p53 function, and is currently employed for the detection of tumor-cells in the urine using fluorescence in-situ hybridisation and microsatellite analysis [7]. Primary tumors and subsequent recurrences are usually of clonal origin. Evaluation of the CI of recurrences may thus gain insight into the malignant potential of field changes.

CI can possibly predict the risk of progression. Richter et al [8] studied 54 stage T1 bladder cancers by conventional comparative genomic hybridisation (CGH). This study suggested that multiple chromosomal losses, as well as copy number changes at certain genomic regions, herald a shorter progression free survival and thus a worse prognosis [8]. Recent advances in microarray technology made it possible to screen the genome for chromosomal imbalances (= loss of heterozygosity (LOH) and/or DNA copy number (CN) alterations) with high resolution [9-13]. Single nucleotide polymorphism (SNP) microarrays offer the opportunity to study $\mathrm{LOH}$ and $\mathrm{CN}$ changes simultaneously [14]. We previously studied chromosomal instability during the progression of bladder cancer using SNP microarrays [10]. In this paper, we aimed at a genomewide investigation of inherent chromosomal alterations related to the risk of progression in bladder cancer. We present an extended material of 48 mostly high-risk nonmuscle invasive bladder cancers with special reference to subsequent progression. Furthermore, we performed CN analyses of 15 most frequently changed gene-loci using quantitative polymerase chain reaction (QPCR) in 77 independent test tumors for validating the association of the changes with the risk of progression of non-muscle invasive bladder cancer.

\section{Methods \\ Study design, patient selection and follow-up}

This case-control study was based on a tissue bank with samples from consecutive bladder cancer patients, treated at our institution since 1994. Patients were followed prospectively. The study was approved by the scientific ethical committee of the county of Aarhus, and all patients gave their informed written consent.

Bladder tumor specimens were cleaved immediately after transurethral resection. One half was analysed by conventional histopathology, the rest snap-frozen in liquid nitrogen and stored at $-80^{\circ} \mathrm{C}$. Tumors were staged according to TNM and graded according to the Bergkvist-classification [15] on a routine base.

We previously analysed tumors from 19 patients from this cohort using Affymetrix ${ }^{\circledR}$ Early Access GeneChip ${ }^{\circledR}$ Mapping 10 K SNP microarrays (Affymetrix, Santa Clara, CA) $[10,16]$. To study the impact of chromosomal alterations on the risk of future disease progression, we extended this dataset by retrospectively selecting further 106 patients from the cohort to total 125 patients. Selection criteria, besides the availability of material, were: non-muscle invasive disease (no previous muscle invasion), a full clinical follow-up without radical treatment (at least five years), and a disease course with "high-risk" tumors (stage $\mathrm{T} 1$ or grade 3 ) or progression (to muscle invasion or metastasis). See Additional File 1 for clinical data of patients and tumors.

Primary tumors from 59 patients were available. Most of these tumors were "high-risk" (stage T1 or grade 3); however, we included 4 primary stage Ta grade 2 tumors with later progression ("high-risk in retrospect") (Table 1). The aim was to investigate inherent chromosomal alterations related to the risk of progression in bladder cancer.

To investigate whether these changes also were present in recurrences, i.e. in field disease, we examined non-primary tumors from 66 different patients. All but three were followed for a previous "high-risk" tumor, but fifteen of the non-primary tumors were "low-risk" themselves (stage Ta grade 2) (Table 1). Non-primary and primary tumors of this study were independent, i.e. from different patients. 
Table I: Stage and grade distribution of the study material of I 25 non-muscle invasive bladder cancers.

\begin{tabular}{|c|c|c|c|c|c|c|c|c|c|c|c|}
\hline & & \multicolumn{5}{|c|}{ Tumors with later progression } & \multicolumn{4}{|c|}{ Tumors with no progression } & \multirow[t]{2}{*}{ Totals } \\
\hline \multicolumn{2}{|c|}{ Stage/Grade } & $\mathrm{TaG}_{2} *$ & $\mathrm{TaG} 2$ & $\mathrm{TaG} 3 / \mathrm{CIS}$ & TIG2 & TIG3 & $\mathrm{TaG} 2$ & $\mathrm{TaG} 3 / \mathrm{CIS}$ & TIG2 & TIG3 & \\
\hline \multirow[t]{2}{*}{ Primary tumors } & SNP microarray & 1 & & & 1 & 10 & & & & 10 & 22 \\
\hline & QPCR only & 3 & & 1 & I & 10 & & & 6 & 16 & 37 \\
\hline \multicolumn{2}{|c|}{ Total primary tumors } & 4 & & 1 & 2 & 20 & & & 6 & 26 & 59 \\
\hline \multirow[t]{2}{*}{ Secondary tumors } & SNP microarray & 1 & 2 & 6 & & 6 & 2 & 4 & 1 & 4 & 26 \\
\hline & QPCR only & 2 & 4 & 9 & & 10 & 4 & 5 & 2 & 4 & 40 \\
\hline \multicolumn{2}{|c|}{ Total secondary tumors } & 3 & 6 & 15 & 0 & 16 & 6 & 9 & 3 & 8 & 66 \\
\hline \multicolumn{2}{|c|}{ Stage distribution (totals) } & 7 & 6 & 16 & 2 & 36 & 6 & 9 & 6 & 26 & 125 \\
\hline \multicolumn{2}{|c|}{ Mean age (range) } & \multicolumn{4}{|c|}{$72.4(54-85)$} & \multicolumn{5}{|c|}{$68.4(46-83)$} & \\
\hline \multirow[t]{2}{*}{ Gender } & Male & & & 54 & & & & 44 & & & 98 \\
\hline & Female & & & 13 & & & & 14 & & & 27 \\
\hline \multicolumn{2}{|c|}{ Totals } & & & 67 & & & & 58 & & & 125 \\
\hline
\end{tabular}

Distribution into primary and secondary tumors, as well as the type of analysis performed, is indicated. Seven low-risk cases with later progression were included and marked with asterisk*. All other tumors were high-risk, or recurrence after high-risk tumors. Detailed clinical information about all individual cases including primary and current stage, grade, treatment, CIS-status, progression free survival, metastases, overall survival and causes of death are listed in Additional File I.

Preselected site biopsies were taken to diagnose concomitant CIS. No adjuvant intravesical chemotherapy was given. Patients were followed prospectively in a routine schedule. Disease recurrences were treated in a standardized fashion. An induction course of intravesical Bacille Calmette-Guerin instillations was applied in 30 cases (CIS, multiple tumors, high recurrence rate), but was not standard treatment at that time. The progression-free survival time was censored at the time of the last control cystoscopy with no evidence of disease. Detailed clinical data are available in Additional File 1.

\section{DNA-purification and SNP microarray analysis}

DNA from frozen tumor tissue and corresponding genomic DNA from blood leucocytes was extracted and purified using a standard DNA-extraction kit (Gentra, Minneapolis, MN). Tumor tissue was trimmed in the microscope to ensure at least $75 \%$ tumor cells. Previously purified DNA (kept at -20 to $-80^{\circ} \mathrm{C}$ ) was used in some cases, having been extracted using various methods, including phenol and ethanol extraction. Concentration and OD ratios were determined using a spectrophotometer.

For high-resolution genome screening, we expanded the previously analysed $10 \mathrm{~K} \mathrm{SNP}$ microarray dataset of 19 tumors [16] with further 29 tumors to total 48 tumors. A selection was made to achieve a homogeneous dataset with respect to the distribution of primary/recurrent, stage $\mathrm{T} 1 /$ stage $\mathrm{Ta}$, and progression/no progression; otherwise it was random. Due to technological progress, the Early Access $10 \mathrm{~K}$ SNP microarray was no longer available; instead we used contemporary Affymetrix Xba GeneChip ${ }^{\circledast}$ Mapping $50 \mathrm{~K}$ SNP microarrays. Analyses of tumor and corresponding blood-DNA were performed as prescribed by the manufacturer. The method was similar for both array types, however, the analyses were performed independently of each other. In brief, 250 ng of DNA was digested using Xba I restriction enzyme, ligated to an Xba adaptor and amplified by PCR using adaptor-specific primers. After purification, $40 \mu \mathrm{g}$ of the PCR products were fragmented using DNase I, labelled, and hybridised to the microarrays at $48^{\circ} \mathrm{C}$ for 18 hours. After hybridisation, arrays were washed 2 times, stained with streptavidin-phycoerythin, washed, linked to biotinylated antistreptavidin antibodies, stained again, and finally washed, followed by reading in a laser scanner. We used an automated Fluidics ${ }^{\circledast}$ station and the GCOS ${ }^{\circledast}$ software provided by the manufacturer.

\section{$\mathrm{LOH}$-analysis}

$10 \mathrm{~K}$ and $50 \mathrm{~K}$ SNP array data were normalized and analysed independently, using empirical means and standard deviations (sd) of 113 [10 K SNP arrays] and 67 [50 K SNP arrays] normal DNA samples extracted from blood leucocytes, respectively, as described [16]. To obtain allelic calls for tumor DNA and the corresponding normal genomic DNA, we used the GDAS ${ }^{\circledast}$ genotyping software supplied by the array manufacturer. Subsequently, a Hidden Markov Model was applied to infer the probability $a_{i j}$ of allelic imbalance/loss of heterozygosity ( $\mathrm{LOH}$ ) for each SNP $j$ on each tumor array $i$ compared to the corresponding array of normal DNA, using the DChip software. For details, see http://biosun1.harvard.edu/complab/dchip/ 
snp.htm, and [17]. For the calculation of the fraction of the genome altered (FGA [LOH]), a probability $a>0.2$ was considered as LOH for both array types.

\section{Copy number analysis}

$10 \mathrm{~K}$ and $50 \mathrm{~K} \mathrm{SNP}$ array data were normalized and analysed separately, as mentioned above. The arrays were normalized and signal values for the individual SNPs were extracted using the DChip software [14]. Briefly, an invariant set normalization method was applied to normalize all arrays of one type at the probe intensity level to a baseline array. After normalization, the perfect match/mismatch difference model was applied to extract a single signal value for each SNP on each array.

Data was then normalized SNP-wise to have mean zero and SD one, using the empirical mean and SD from the respective normal arrays, $z_{i j}=\left(y_{i j}-m_{j}\right) / s_{j}$, where $y_{i j}$ is the signal value for array $i$ and SNP $j, \mathrm{~m}_{j}$ is the mean of SNP $j$ and $s_{j}$ the SD of SNP $j$. Data $z_{i j}$ was further smoothened over $k$ SNPs to obtain $x_{i j}=\Sigma z_{i k} / k$, where the sum is over $k$ $=j-K, \ldots, j+K$. Here $K=5$ for both $10 \mathrm{~K}$ and $50 \mathrm{~K}$ arrays. For the calculation of the fraction of the genome altered (FGA $[\mathrm{CN}])$, signal values $x$ higher than 3 or lower than -3 (mean +/-3 sd, corresponding to probability $p$ of 1.degree's error < 0.01) were considered copy number imbalanced.

\section{Creation of common SNP dataset}

A total of 6,400 common probesets were identified on both microarrays, using the annotation-files provided by the array-manufacturer. Data $a$ (LOH probability) and $x$ (standardised signal value) for these SNPs were extracted from both the $10 \mathrm{~K}$ and $50 \mathrm{~K}$ datasets and assigned to unique reference-SNP-IDs http://www.ncbi.nlm.nih.gov/

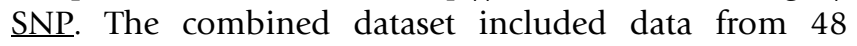
patients, 19 analysed on $10 \mathrm{~K}$ arrays and 29 analysed on $50 \mathrm{~K}$ arrays. LOH data was missing for one $50 \mathrm{~K}$ array (\#83) because of unmatched blood-DNA, and signal intensity data were lost for two $10 \mathrm{~K}$ arrays (\#1013-1 and $\# 1033-2)$ after extraction of the allelic calls.

The overall median FGA (both FGA [LOH] and FGA [CN]) was $5 \%$, which was chosen as the cut-off value in the survival analyses.

Test for differences in chromosome alterations between progressing and non-progressing tumors

The average of $x_{i j}$ was calculated for tumors in the 2 groups (progressors and non-progressors) for each SNP, and the difference $d_{j}$ between the two averages was found. High and low values of $\mathrm{d}_{j}$ were indicative of group differences. Low values indicated that group 1 had higher mean than group 2, and high values that group 2 had higher mean. Long segments of SNPs with high (or low) values of $\mathrm{d}_{j}$ were more likely to be due to true differences; thus the seg- ment lengths were also calculated. Significance of the observed statistics was calculated using a permutation test: New groups of the same sizes were formed by permuting group labels 50,000 times and the number of times (in \%) a value higher (or lower) was observed, was calculated. This test was performed using the combined dataset (see Additional Files 2 and 3) and using the $50 \mathrm{~K}$ dataset alone, because of the higher resolution of this dataset (see Additional Files 4 and 5).

\section{Copy number analysis using QPCR}

Fifteen unstable chromosomal regions were identified using the SNP microarray data (see Additional Files 2, 3, 4 and 5). Within these regions, we selected 15 gene loci of documented or putative bladder cancer relevance (Table 2). To determine relative DNA copy number changes of these loci, we performed QPCR using an ABI Prism $7500^{\circledR}$ Real time PCR cycler (Applied Biosystems, Foster City, CA) with standard QPCR conditions according to the manufacturer's instructions (all analyses were performed in triplicate). Primer sequences are listed in Additional File 6. Standard curves were constructed using pooled normal DNA from blood.

We successfully analysed 101 tumors, including 25 tumors analysed on $50 \mathrm{~K}$ SNP microarrays. The obtained quantity values were standardised to mean one by division with the median quantity value of the 15 examined probes (the median value was considered to be most likely to represent a normal copy number). Results were $\log$ transformed. Normal variation was estimated using tumors that were stable on $50 \mathrm{~K}$ microarrays $(\mathrm{FGA}<0.05$ ) as standard. Quantity values exceeding two times the normal variation were considered copy number imbalanced. We used the STATA 8.0 statistical software package (StataCorp LP, College Station, TX).

\section{Results}

In total, 67 patients (54\%) suffered disease progression, which was defined as occurrence of muscle invasion (stage T2-4) or metastatic disease (stage N2-3 or stage M1). The high progression rate is due to the study design (exclusion of radically treated patients and patients with no progression but incomplete follow-up). Fifty-eight patients showed no evidence of progression. Follow-up was median 80 months. The median time to progression was 17 months. Nine patients had longer than five years progression-free survival, with late progression. We found CIS concomitant to 38 tumors (31\%), with equal distribution between progressors and non-progressors in this high-risk material (Table 1).

\section{SNP microarrays ( $n=48)$ : Fraction of the genome altered (FGA)}

The dataset was composed of 22 primary tumors (hereof 21 stage T1), and 26 recurrent tumors (hereof 15 stage 
Table 2: Selected gene loci, gene IDs, cytoband and genomic annotation of the amplicons used for QPCR validation.

\begin{tabular}{|c|c|c|c|c|}
\hline Gene & Gene-ID & Cytoband & Amplicon position (NCBI Build 35.I [hg I7]) & Amplicon position (hg I 8) \\
\hline SIOOA8 & 6279 & $|q 2|$ & chrl:|50,|75,956-|50,|76,07| & chrl:151,629,507-151,629,622 \\
\hline FNI & 2335 & $2 q 34$ & chr2:216,II2,380-216, I I2,466 & chr2:215,995,1। $19-215,995,205$ \\
\hline RAFI & 5894 & $3 p 25$ & chr3:| 2,635,039-12,635, |48 & Identical \\
\hline$D A B 2$ & 1601 & $5 p 13$ & $\operatorname{chr} 5: 39,4 \mid 8,493-39,418,602$ & identical \\
\hline CSPG2 & 1462 & $5 q \mid 4.3$ & $\operatorname{chr} 5: 82,821,732-82,821,832$ & identical \\
\hline E2F3 & |87| & $6 p 22$ & chr6:20,598,4l0-20,598,5I6 & identical \\
\hline SFRPI & 6422 & $8 p|2-p| 1.1$ & chr8:4I,285,606-4I,285,686 & identical \\
\hline TUSC3 & 7991 & $8 p 22$ & chr8:|5,524,970-|5,525,08| & identical \\
\hline EDDI & 51366 & $8 q 22$ & chr8: $103,366,989-103,367,080$ & identical \\
\hline MYC & 4609 & $8 q 24.12-q 24.13$ & chr8: |28,822, I 58-|28,822,287 & identical \\
\hline CDKN2A & 1029 & $9 p 21$ & chr9:21,957,845-21,957,973 & identical \\
\hline KLF4 & 9314 & $9 q 31$ & chr9:107,331,II7-107,331,240 & chr9:109,291,383-109,291,506 \\
\hline MDM2 & 4193 & $12 q 14.3-q 15$ & $\operatorname{chr}|2: 67,5| 9,75|-67,5| 9,858$ & Identical \\
\hline$R B I$ & 5925 & $13 q \mid 4.2$ & $\operatorname{chr} \mid 3: 47,853,426-47,853,564$ & Identical \\
\hline TP53 & 7157 & $17 p \mid 3.1$ & $\operatorname{chr}|7: 7,520,09|-7,520,236$ & Identical \\
\hline
\end{tabular}

Loci were selected based on the frequency of alterations in the total SNP material, and on bladder cancer relevance according to literature and our own gene expression analyses [22]. Loci are not referring to the (insignificant) relative changes characterizing progressing tumors listed on Table 3 , except SFRPI (8pI I). Primer sets leading to amplicons of 80-120 basepairs were designed using the Primer3 software http://frodo.wi.mit.edu/cgibin/primer3/primer3.cgi. The QPCR primer sequences are listed in Additional File 6.

T1). 27 patients had progressing disease, while 21 had no progression (see Additional File 1 for details). The probability of later progression was not associated with the fraction of the genome altered (FGA). Figure 1 illustrates the progression-free survival, dependent on whether the examined tumor was stable or unstable. Results of all tumors (corrected for stage) and separately for stage T1 tumors are shown. Figure 2 shows the relative significance of the FGA and its variation, dependent on whether later progression occurred or not. The FGA $[\mathrm{CN}]$ and FGA $[\mathrm{LOH}]$ for all individual tumors is listed in Additional File 7.

In primary tumors separately $(\mathrm{n}=22$, hereof 21 stage $\mathrm{T} 1)$ we found no difference between tumors with and with no later progression ( $\mathrm{p}=0.62$ and $\mathrm{p}=0.2$, for FGA(LOH) and FGA(CN), respectively; Mann-Whitney ranksum tests). Recurrent lesions ( $\mathrm{n}=26$, stage T1: $\mathrm{n}=15)$, representing underlying field disease, even had a slight tendency towards more stable tumors among those with later progression ( $\mathrm{p}=0.08$ and $\mathrm{p}=0.52$; corrected for T-stage). Equivalent results were obtained using Kaplan-Meier-estimates of progression-free survival time (stable vs. unstable). Cox' regression analyses, taking into account both the time to progression and the relative significance of FGA (in categories), did neither reveal any significant differences.

In general, the FGA was significantly higher for stage T1 tumors ( $p=0.004$ and $p=0.0005)$ and grade 3 tumors ( $p$ $=0.03$ and $p=0.003$ ) compared to stage Ta and grade 2 tumors, respectively. The FGA was also positively correlated to the occurrence of CIS. Notably, eleven tumors $(23 \%)$ had very few alterations (FGA $<0.01)$. Interestingly, seven of these tumors were stage $\mathrm{T} 1$, and the disease progressed in seven of these cases (four stage T1 tumors).

\subsection{Test for differences in chromosomal alterations related to subsequent progression}

This test was performed to reveal specific chromosomal areas related to subsequent progression. However, the tumors showed wide chromosomal heterogeneity, which resulted in a considerable risk of detecting "significance by chance", supposed the relatively low number of samples $(n=48)$ compared to the number of variables $(n=$ 6,400 ). We employed the fact that all SNPs are mutually dependent through their mapped positions: a number of neighbouring "significant" SNPs altered in the same way were more likely to represent true changes (segment analysis). Genome-wide differences are shown in Additional Files 2 and 3 (Combined dataset, $\mathrm{CN}$ and $\mathrm{LOH}$ differences, respectively), and with the higher resolution of the $50 \mathrm{~K}$ dataset in Additional Files 4 and 5.

Using a combined approach of LOH and copy number analysis with restriction to changes more pronounced in progressing tumors (and thus independent of stage), we identified some areas which may play a role in the progression of bladder cancer (Table 3). Most prominent was loss/LOH of chromosome 8p11-p12 (35-43 Mb). To reveal statistic significance of these segments, we performed a permutation analysis. However, none of the 

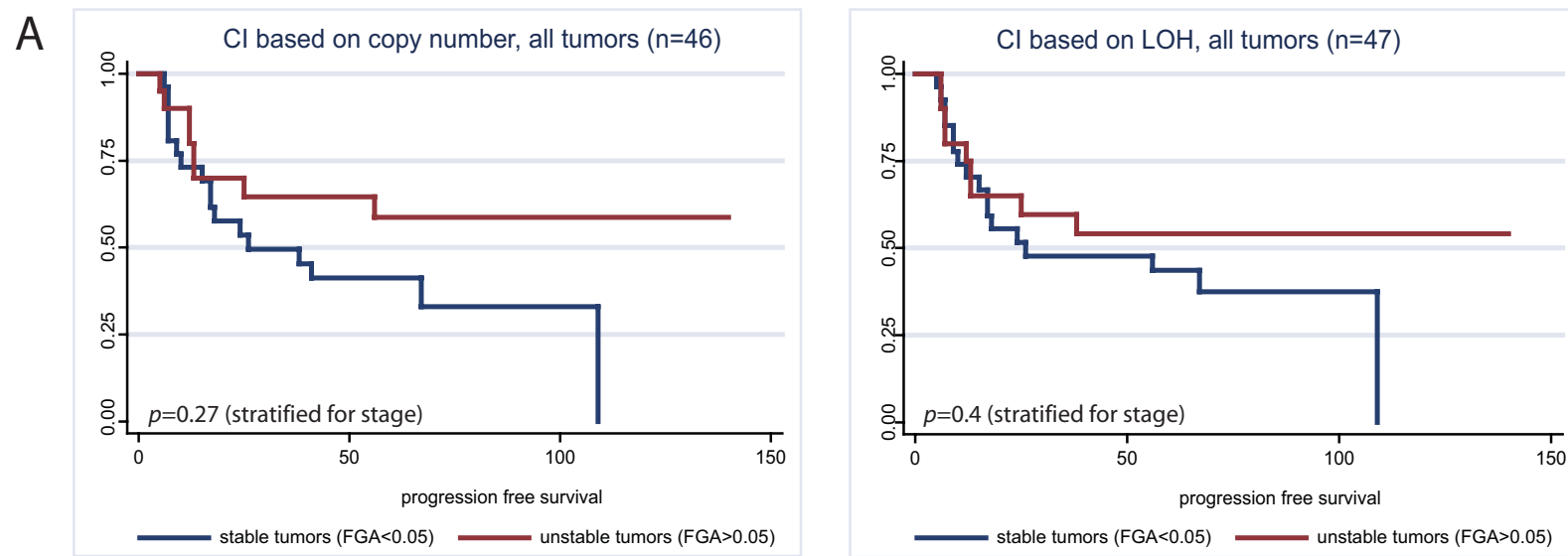

B
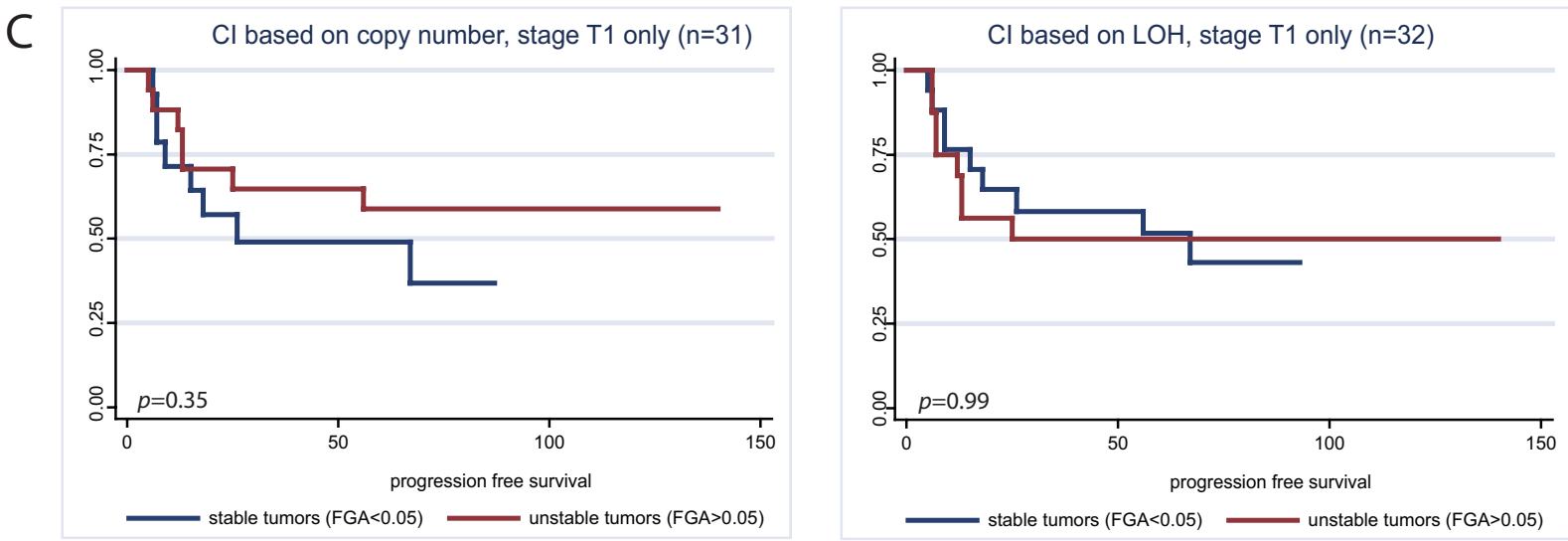

\section{Figure I}

Kaplan Meier estimates of progression-free survival according to chromosomal instability (CI). Tumors are divided in stable and unstable using a cut-off for the fraction of the genome altered (FGA) $>0.05$. Here, the influence of the time to progression, not the relative size of the FGA is analysed. Logrank-tests for differences between progressing and nonprogressing tumors showed no significant difference. A: all tumors, $\mathrm{Cl}$ based on copy number changes $(n=46)$. B: all tumors, $\mathrm{Cl}$ based on LOH $(n=47)$. C: Stage TI tumors only, Cl based on copy number changes $(n=32)$. D: Stage TI tumors only, Cl based on $\mathrm{LOH}(\mathrm{n}=33)$.

identified areas was significant in segment analysis (Table 3).

\subsection{Copy number analysis of I5 selected gene loci using QPCR (I0I tumors, Figure 3)}

A semi quantitative illustration of the results in all tumors is given on Figure 3. We confirmed that stage T1 tumors had significantly more alterations than stage Ta tumors ( $p$ $=0.008$, Mann-Whitney-test). In congruence with the microarray results, there was no correlation between the number of alterations and subsequent progression. QPCR data showed good association with the data obtained from $50 \mathrm{~K}$ microarrays $(r=0.67)$. However, no correlation between any of the markers and the risk of progression was found, including the SFRP1 marker (8p11-p12). Neither did we find significant influence of any confounder studied, including age, gender, multiplicity, primary/secondary, concomitant CIS, and BCG-treatment, in multivariate analyses. The number of changed markers for all individual tumors is listed in Additional File 7.

\section{Discussion}

The aim of the present study was to investigate inherent chromosomal changes related to the risk of progression in bladder cancer, as well as chromosomal instability (CI)/ fraction of the genome altered (FGA) as a prognostic marker. A previous CGH study of 54 stage T1 tumors suggested a shorter progression free survival of tumors with multiple copy number losses (CI) or alterations at certain regions [8]. We utilised SNP microarrays to perform highresolution genome-wide combined LOH and copy number screening of 21 primary stage $\mathrm{T} 1$ tumors. We 


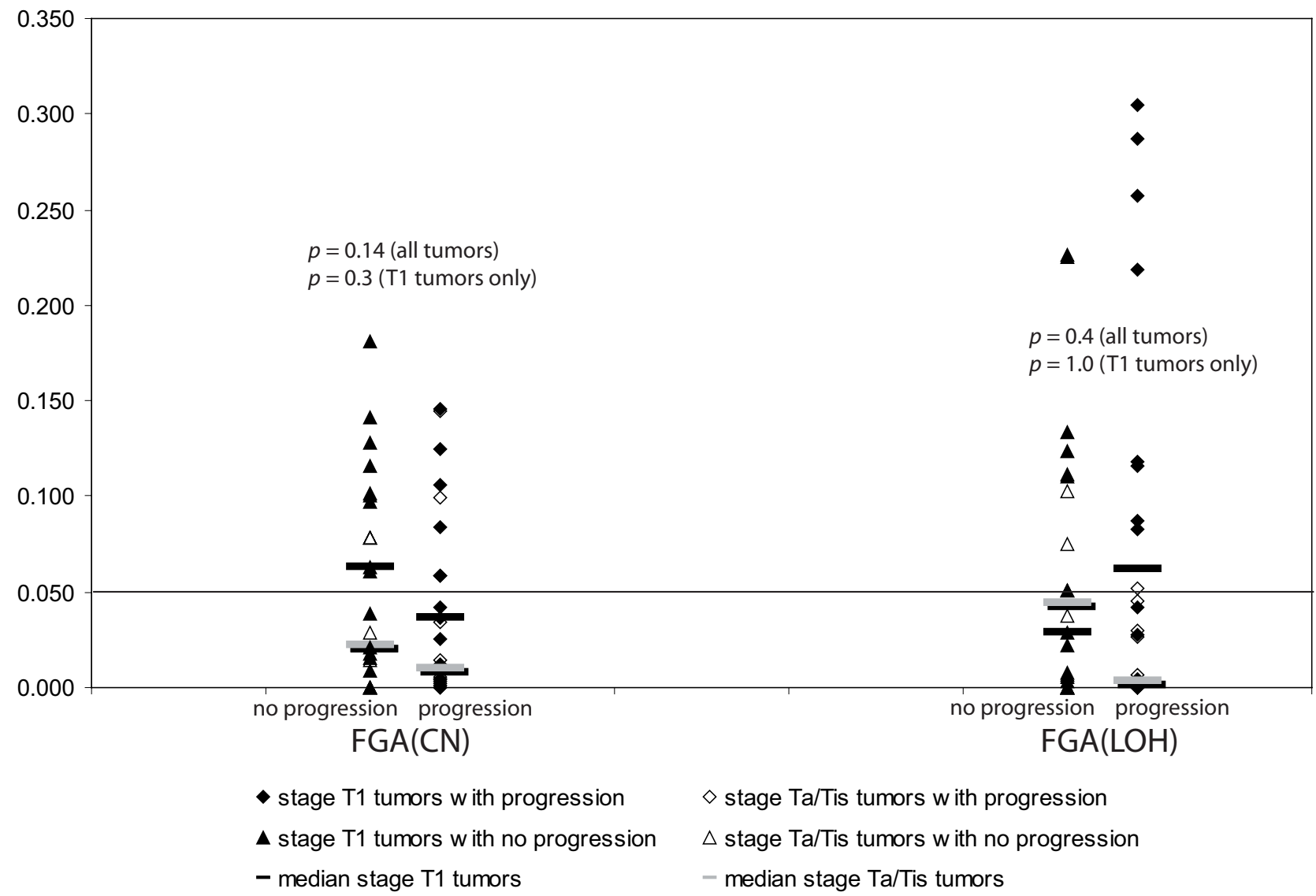

\section{Figure 2}

The fraction of the genome altered (FGA) against the clinical course (progression/no progression), separately for copy number changes (left) and LOH (right). Non-progressing tumors in triangles, progressing in diamonds. Solid symbols indicate stage TI tumors. Ranksum-tests for difference in FGA between progressing and non-progressing tumors for all tumors $(n=48)$ and for stage TI tumors only $(n=33)$ showed no significant difference. Here, the relative size of the FGA, not the influence of the time to progression is analysed.

demonstrated frequent chromosomal changes in most of these tumors. However, we were not able to show any association between chromosomal changes and subsequent progression. One explanation may be that stage T1 tumors can be completely resected. Under this condition, the future course is not determined by the malignant potential of the resected tumor, but the residual tumor cells in the bladder.

Recurrent tumors are usually the result of the spread of a single tumor clone in the urothelium. Only a minority has been shown to be of different clonal origin, i.e. new primary tumors [18]. We analysed 26 recurrent tumors, to determine whether residual tumor cells in the bladder already harbour changes related to the risk of progression, because these changes should be present in all recurrences as well. Our results demonstrated less frequent chromosomal alterations in non-invasive recurrences (stage Ta), even if the primary lesion was stage $\mathrm{T} 1$, and the disease later progressed. Recurrent stage T1 tumors had as much alterations as the primary stage T1 tumors; however, this was again independent of later progression. These results indicated that the precursor lesions of recurrent tumors also those with later progression - had relatively few alterations, i.e. they were apparently chromosomal stable. The frequent alterations seen in stage $\mathrm{T} 1$ (and muscle-invasive) tumors were then the results of (more or less) stochastic events during the development of the individual tumor, and not inherent characteristics of field changes with high malignant potential. These findings are well inline with the "field-first-tumor-later" concept recently developed by Höglund [19], according to which recurring 
Table 3: Candidate areas with chromosomal alterations characterizing non-muscle invasive bladder cancers with subsequent progression.

\begin{tabular}{|c|c|c|c|c|c|c|}
\hline Chromosome & Cytoband & $\begin{array}{l}\text { Physical position } \\
\text { (hg|7/NCBI build } 35.1 \text { ) }\end{array}$ & $\begin{array}{l}\text { chromosomal changes of } \\
\text { progressing tumors }\end{array}$ & $\begin{array}{l}\text { Candidate genes } \\
\text { (selection) }\end{array}$ & $p$ (single SNPs) & $p$ (segment) \\
\hline 1 & p31.1-p22.3 & $82-86 \mathrm{Mb}$ & $\mathrm{CN}$ gain & BCLIO & 0.003 & n.s. \\
\hline 2 & $\mathrm{q} 33.3-\mathrm{q} 34$ & $208-213 \mathrm{Mb}$ & $\mathrm{CN}$ gain & CREBI, MAP2 & 0.001 & 0.05 \\
\hline 3 & p21-p|4.3 & $42-64 \mathrm{Mb}$ & $\mathrm{CN}$ gain & CCR-cluster & 0.01 & n.s. \\
\hline 4 & $\mathrm{pl3}$ & $40-45 \mathrm{Mb}$ & $\mathrm{CN}$ loss/LOH & & 0.02 & n.s. \\
\hline 6 & $q 15-q 23.2$ & $89-133 \mathrm{Mb}$ & $\mathrm{CN}$ loss/LOH & TPD52LI & 0.01 & n.s. \\
\hline 8 & pl2-p|l.2I & $33 M-43 M b$ & $\mathrm{CN}$ loss/LOH & SFRPI, TACCI, FGFRI & 0.01 & n.s. \\
\hline 10 & pl5.I-p|4 & $3.5 \mathrm{M}-13 \mathrm{Mb}$ & $\mathrm{CN}$ gain & $\begin{array}{l}\text { GATA3, NETI, PKCQ, } \\
\text { ILI 5RA }\end{array}$ & 0.005 & n.s. \\
\hline 10 & pll & $30-36 \mathrm{Mb}$ & $\mathrm{CN}$ gain & & 0.005 & n.s. \\
\hline 15 & $\mathrm{q} 25.3$ & $84-85 \mathrm{Mb}$ & $\mathrm{CN}$ loss & & 0.01 & n.s. \\
\hline
\end{tabular}

SNP microarray analysis (significance $p=$ probability of detecting difference between progressing and non-progressing tumors by chance, permutation analysis). A survey of the entire genome is possible in Additional Files 2, 3, 4 and 5.

tumors originate from a shared field of premalignant cells (with relatively few chromosomal changes) and not from previous overt tumors. Consequently, it was impossible to predict the future course of a disease from chromosomal changes of a marker lesion, given that this lesion was completely resected. Notably, about $25 \%$ of the tumors showed virtually no CI. This was independent of stage, concomitant CIS, and future progression. In conclusion, with the huge variation of chromosomal changes demonstrated, chromosomal alterations are unlikely to play a role as a predictive instrument in non-muscle invasive bladder cancer - at least with the resolution provided here, which is higher than in the previous report [8].

The same considerations also apply with respect to specific chromosomal alterations. The paper of Richter et al. [8] suggested several specific chromosomal changes that may have significance for the malignant development independent of general CI. Unfortunately, we were not able to confirm these results. The identification of significant areas among many possible areas is challenging, given the variation of chromosomal changes observed. If the number of possible areas (here: SNPs) by far exceeds the number of samples, conventional statistical methods do not perform well. Correction for multiple comparisons is mandatory. In our segment analysis, we employed the dependency of the SNPs on their mapped positions to reduce the number of variables from 6,400 (SNPs) to 22 (chromosomes). This permutation analysis reveals the probability of finding a segment of significant SNPs of a given length on a given chromosome by chance. However, this analysis must fail to identify small significant segments, particularly on chromosomes with common largescale alterations, e.g. chromosome 8.

We had the intention to validate the predictive potential of specific chromosomal areas using QPCR-based copy- number analysis. Since no significant areas could be documented (Table 3), we decided to evaluate general CI using areas with frequent instability (see Additional Files 2, 3, 4 and 5) and possible bladder cancer relevance, according to the literature (including [8]) and our own gene expression analyses. We did not find any correlation between the risk of progression and any of the chosen markers, or with the number of changed markers/CI. These results were in line with the SNP-data. However, the area on chromosome $8 \mathrm{p} 11$ deserves special attention. Not only did we pinpoint the area as significant in SNP analyses (Table 3 ), it has also previously been stressed as being very unstable not only in bladder cancer, but in many other solid cancers [20]. The area has been reported to be frequently involved in chromosomal rearrangements [20]. Candidate genes in this region include SFRP1. Chromosomal loss and reduced expression of the mRNA and the SFRP1 protein, an antagonist of Wnt-signalling, have been reported by several other groups to be associated with bladder cancer progression [21], and we found the same in our own gene expression profiles [22]. In QPCR analysis, the SFRP1 marker was very unstable; however, this was not related to progression free survival. The clinical significance of this region still remains to be elucidated.

We adjusted for the grade of dysplasia, an important risk factor for the clinical risk estimation, using the Bergkvist grading system under routine conditions [15]. This system has been in routine use all over Scandinavia for many years, but is uncommon in the rest of the world. It can be translated into the $1999 \mathrm{WHO} / \mathrm{ISUP}$ grading system [23], according to which most of our tumors would be classified as "high grade". A pathological review of the slides would have been optimal, but was not performed. However, we considered the grading system of minor importance, as long as the analysis is adjusted for this factor. 


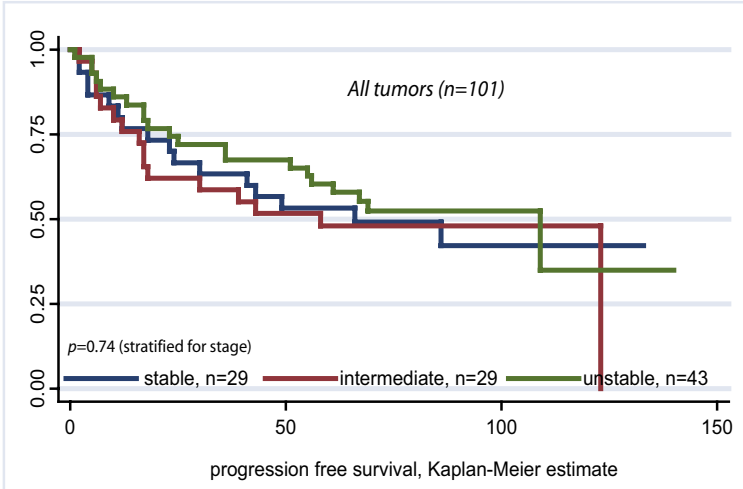

A

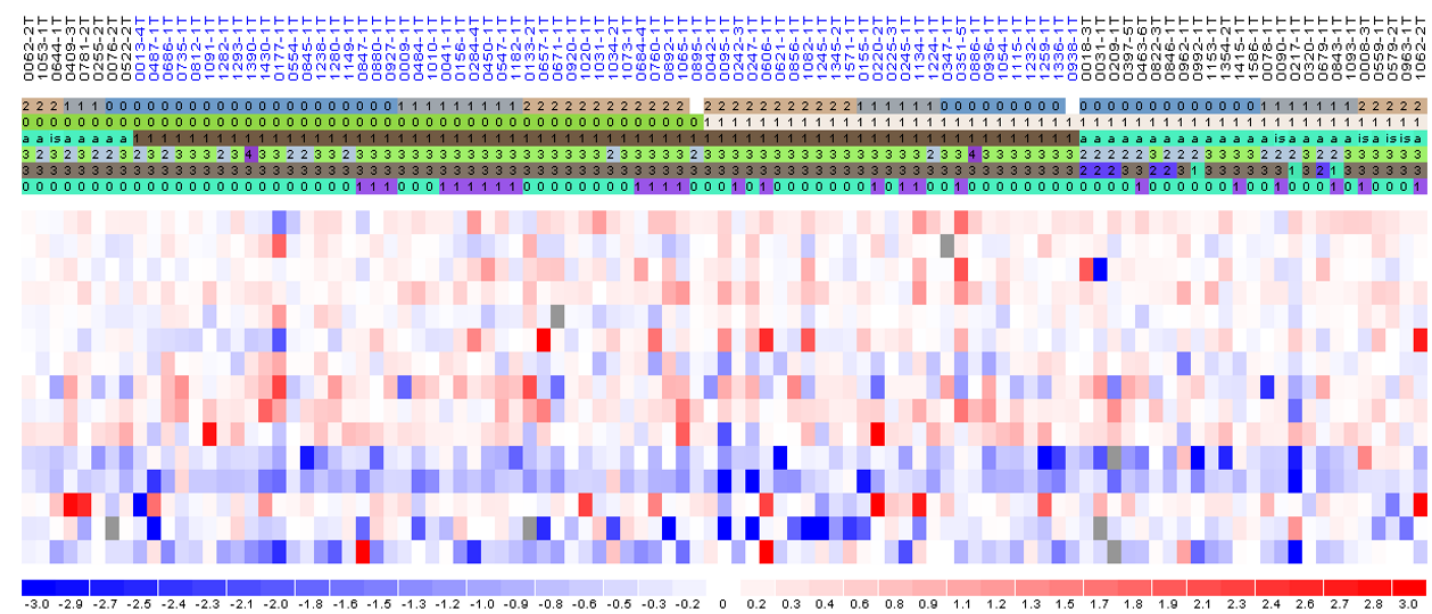

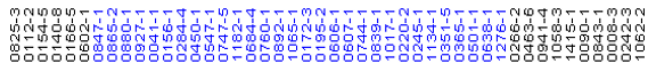

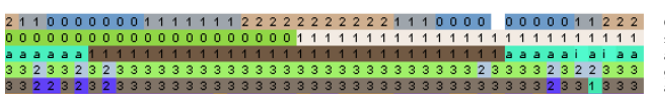

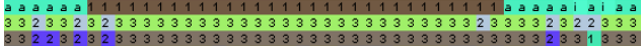

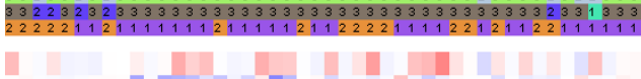

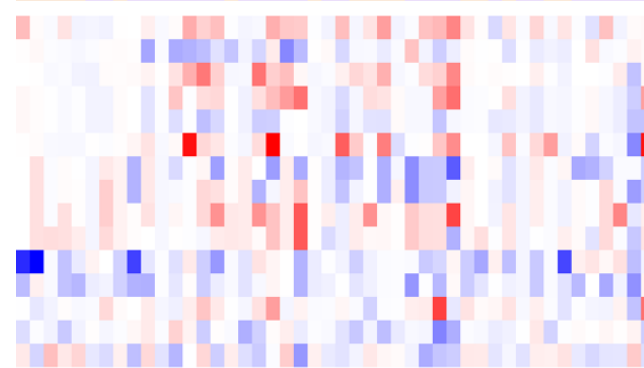

grade of dysplasia

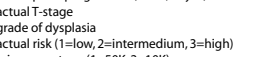
S100A8 (1q21) FN1 (2q34) RAF1 (3p25) RAF1 (3p25) DAB2 (5p13) CSPG2 (5q14.3) E2F3 (6p22) TUSC3 (8p22) SFRP1 (8p12-p11.1) EDD1 (8q22) MYC (8q24.12-q24.13) CDKN2A (9p21) KLF4 (9q31) MDM2 (12q14.3-q15) RB1 (13q14.2) TP53 (17p13.1)

\section{Figure 3}

Copy number analysis of 15 marker regions (see Table 2). A: Kaplan Meier estimates of progression free survival according to number of copy-number imbalanced marker regions in QPCR analysis. Stable (0-2 changes); intermediate (3-4 changes), unstable (> 4 changes). B: Copy number alterations (log2 ratios) of candidate regions determined by SNP microarrays (SNPs flanking the marker regions). Tumors in columns, marker regions in rows. Top row indicates patient and tumor ID, bars below refer to tumor characteristics. Non-progressors to the left, progressors to the right; stage TI tumors in the middle, stage Ta tumors on the flanks. Tumors are further sorted according to their CIS status: Tumors with concomitant CIS in the very center and on the very verges, tumors with later or no CIS in between. Furthermore, the grade of dysplasia (Bergkvist[ [5]), the actual clinical risk (including previous history) [3] and the used microarray $(I=50 \mathrm{~K}, 2=10 \mathrm{~K}$ ) are indicated. Data area: Red: Copy number gain. Blue: Copy number loss. C: Copy number analysis of the same candidate regions by QPCR of $\mathrm{IOI}$ tumors, hereof 77 independent tumors. Tumors are sorted in the same way as in B. A survey of the number of altered regions is provided in Additional File 7.

\section{Conclusion}

Chromosomal instability did not characterise tumors with subsequent progression. Our results suggest that chromosomal instability develops during the progression process, and is rare in precursor lesions, as indicated by the less pronounced chromosomal changes in recurrent tumors. Chromosomal alterations, particularly losses, of chromsome 8p11 (34-43 Mb) may be present in field disease, and may have a role in its malignant development.

\section{Abbreviations}

(CIS): Carcinoma in situ of the urinary bladder; (DNA):desoxyribonucleic acid; (SNP): single nucleotide polymorphism; (CI): chromosomal instability; (CN): DNA copy number; (LOH): loss of heterozygosity; (FGA): fraction of the genome altered; (QPCR): quantitative polymerase chain reaction.

\section{Competing interests}

The authors declare that they have no competing interests. 


\section{Authors' contributions}

$\mathrm{KZ}$ reviewed the patients' history and pathological data, performed sample selection and retrieval, carried out the molecular analyses, performed data analysis and part of the statistical analyses, interpretation of data, and drafted the manuscript. CW developed and carried out the procession of the microarray data and the statistical analyses. KMEJ was responsible for the sampling of tissue, information of patients, and the clinical database. TFØ participated in the conception and design of the study, and critically revised the manuscript. LD helped with the analysis and interpretation of the data, and helped to draft the manuscript.

\section{Additional material}

\section{Additional file 1}

Clinical data of the patients in the study. Including age and gender, stage and grade of the primary and (if different) analysed tumors, supplementary treatment (BCG), CIS-status, length of follow-up/progressionfree survival, causes of death and overall survival.

Click here for file

[http://www.biomedcentral.com/content/supplementary/14712407-9-149-S1.xls]

\section{Additional file 2}

Genome-wide copy number differences, $50 \mathrm{~K}+10 \mathrm{~K}$ data (6.4 K resolution). Graphical illustration of genome-wide copy number differences between tumors with and with no subsequent progression. Compiled results of all SNP microarray data $(n=46)$ with a resolution of $6.4 \mathrm{~K}$ SNPs.

Click here for file

[http://www.biomedcentral.com/content/supplementary/14712407-9-149-S2.pdf]

\section{Additional file 3}

Genome-wide differences in probability of $\mathrm{LOH}, 50 \mathrm{~K}+10 \mathrm{~K}$ data. Graphical illustration of genome-wide differences in probability of $\mathrm{LOH}$ between tumors with and with no subsequent progression. Compiled results of all SNP microarray data $(n=47)$.

Click here for file

[http://www.biomedcentral.com/content/supplementary/14712407-9-149-S3.pdf]

\section{Additional file 4}

Genome-wide copy number differences, $50 \mathrm{~K}$ data only (41 K resolution). Graphical illustration of genome-wide copy number differences between tumors with and with no subsequent progression. $50 \mathrm{~K}$ SNP microarray data only $(n=29)$.

Click here for file

[http://www.biomedcentral.com/content/supplementary/14712407-9-149-S4.pdf]

\section{Additional file 5}

Genome-wide differences in probability of $\mathrm{LOH}, 50 \mathrm{~K}$ data only. Graphical illustration of genome-wide differences in probability of $\mathrm{LOH}$ between tumors with and with no subsequent progression. $50 \mathrm{~K}$ SNP microarray data only $(n=28)$.

Click here for file

[http://www.biomedcentral.com/content/supplementary/14712407-9-149-S5.pdf]

\section{Additional file 6}

Selected gene loci, primer sequences and genomic annotation of the amplicons used for QPCR validation.

Click here for file

[http://www.biomedcentral.com/content/supplementary/14712407-9-149-S6.doc]

\section{Additional file 7}

Chromosomal imbalance of the analysed tumors. Contains detailed data of the fractions of the genome altered (FGA), specified separately for copy number and LOH changes, as well as the number of QPCR copy number alterations

Click here for file

[http://www.biomedcentral.com/content/supplementary/14712407-9-149-S7.xls]

\section{Acknowledgements}

The present work was funded by the Danish Cancer Research Foundation, the Danish Cancer Society, and the John and Birte Meyer Foundation, which is gratefully acknowledged. We would like to thank the staff at Molecular Diagnostic Laboratory (Department of Molecular Medicine), in particular Pamela Celis and Gitte Høj, for their excellent technical assistance.

\section{References}

I. Herr HW: Natural history of superficial bladder tumors: 10to 20-year follow-up of treated patients. World J Urol 1997, 15:84-88.

2. Zieger $\mathrm{K}$, Olsen PR, Wolf H, Hojgaard K: Long term follow-up of superficial invasive bladder carcinoma with or without concomitant epithelial atypia - recurrence and progression. Scand J Urol Nephrol 2002, 36:52-59.

3. Sylvester RJ, Meijden AP van der, Oosterlinck W, Witjes JA Bouffioux C, Denis L, et al.: Predicting recurrence and progression in individual patients with stage $\mathrm{Ta}$ TI bladder cancer using EORTC risk tables: a combined analysis of 2596 patients from seven EORTC trials. Eur Urol 2006, 49:466-5.

4. Spruck CH III, Ohneseit PF, Gonzalez-Zulueta M, Esrig D, Miyao N, Tsai YC, et al:: Two molecular pathways to transitional cell carcinoma of the bladder. Cancer Res 1994, 54:784-788.

5. Hartmann A, Schlake G, Zaak D, Hungerhuber E, Hofstetter A, Hof staedter F, et al:: Occurrence of chromosome 9 and p53 alterations in multifocal dysplasia and carcinoma in situ of human urinary bladder. Cancer Res 2002, 62:809-8I8.

6. Catto JW, Meuth M, Hamdy FC: Genetic instability and transitional cell carcinoma of the bladder. BJU Int 2004, 93:19-24.

7. van Rhijn BW, Poel HG van der, Kwast $\mathrm{TH}$ van der: Urine markers for bladder cancer surveillance: a systematic review. Eur Urol 2005, 47:736-748.

8. Richter J, Wagner U, Schraml P, Maurer R, Alund G, Knonagel H, et al.: Chromosomal imbalances are associated with a high risk of progression in early invasive (pTI) urinary bladder cancer. Cancer Res 1999, 59:5687-569I.

9. Hoque MO, Lee CC, Cairns P, Schoenberg M, Sidransky D: Genome-wide genetic characterization of bladder cancer: a comparison of high-density single-nucleotide polymorphism arrays and PCR-based microsatellite analysis. Cancer Res 2003, 63:2216-2222.

10. Koed K, Wiuf C, Christensen LL, Wikman FP, Zieger K, Moller K, et al.: High-density single nucleotide polymorphism array defines novel stage and location-dependent allelic imbalances in human bladder tumors. Cancer Res 2005, 65:34-45.

II. Blaveri E, Brewer JL, Roydasgupta R, Fridlyand J, DeVries S, Koppie T, et al.: Bladder cancer stage and outcome by array-based comparative genomic hybridization. Clin Cancer Res 2005, I I:7012-7022. 
12. Veltman JA, Fridlyand J, Pejavar S, Olshen AB, Korkola JE, DeVries S, et al: Array-based comparative genomic hybridization for genome-wide screening of DNA copy number in bladder tumors. Cancer Res 2003, 63:2872-2880.

13. Hurst CD, Fiegler H, Carr P, Williams S, Carter NP, Knowles MA: High-resolution analysis of genomic copy number alterations in bladder cancer by microarray-based comparative genomic hybridization. Oncogene 2004, 23:2250-2263.

14. Zhao X, Li C, Paez JG, Chin K, Janne PA, Chen TH, et al.: An integrated view of copy number and allelic alterations in the cancer genome using single nucleotide polymorphism arrays. Cancer Res 2004, 64:3060-307I.

15. Bergkvist A, Ljungqvist A, Moberger G: Classification of bladder tumours based on the cellular pattern. Preliminary report of a clinical-pathological study of $\mathbf{3 0 0}$ cases with a minimum follow-up of eight years. Acta Chir Scand 1965, 130:37I-378.

16. Zieger K, Dyrskjot L, Wiuf $C$, Jensen JL, Andersen $C L$, Jensen KM, et al.: Role of activating fibroblast growth factor receptor 3 mutations in the development of bladder tumors. Clin Cancer Res 2005, I I:7709-77I9.

17. Lin M, Wei LJ, Sellers WR, Lieberfarb M, Wong WH, Li C: dChipSNP: significance curve and clustering of SNP-array-based loss-of-heterozygosity data. Bioinformatics 2004, 20:1233-1240.

18. Hafner C, Knuechel R, Stoehr R, Hartmann A: Clonality of multifocal urothelial carcinomas: 10 years of molecular genetic studies. Int J Cancer 2002, I 0 I: I-6.

19. Hoglund M: On the origin of syn- and metachronous urothelial carcinomas. Eur Urol 2007, 51:1 185-1193.

20. Adams J, Williams SV, Aveyard JS, Knowles MA: Loss of heterozygosity analysis and DNA copy number measurement on $8 \mathrm{p}$ in bladder cancer reveals two mechanisms of allelic loss. Cancer Res 2005, 65:66-75.

21. Stoehr R, Wissmann C, Suzuki H, Knuechel R, Krieg RC, Klopocki E, et al:: Deletions of chromosome 8p and loss of sFRP I expression are progression markers of papillary bladder cancer. $L a b$ Invest 2004, 84:465-478.

22. Dyrskjot L, Zieger K, Kruhoffer M, Thykjaer T, Jensen JL, Primdahl H, et al: A molecular signature in superficial bladder carcinoma predicts clinical outcome. Clin Cancer Res 2005, I I:4029-4036.

23. Busch C, Algaba F: The WHO/ISUP 1998 and WHO 1999 systems for malignancy grading of bladder cancer. Scientific foundation and translation to one another and previous systems. Virchows Arch 2002, 44I:105-108.

\section{Pre-publication history}

The pre-publication history for this paper can be accessed here:

http://www.biomedcentral.com/1471-2407/9/149/pre

pub

Publish with Biomed Central and every scientist can read your work free of charge

"BioMed Central will be the most significant development for disseminating the results of biomedical research in our lifetime. "

Sir Paul Nurse, Cancer Research UK

Your research papers will be:

- available free of charge to the entire biomedical community

- peer reviewed and published immediately upon acceptance

- cited in PubMed and archived on PubMed Central

- yours - you keep the copyright 